\title{
SPECTROPHOTOMETRY AND KINEMATICS OF THE NEWLY DISCOVERED PN IN THE OUTHER FIELD OF THE LMC
}

\author{
M.A. DOPITA and E. VASSILIADIS \\ Mt. Stromlo and Siding Springs Observatories, Institute of Advanced Studies, The \\ Australian National University, Canberra \\ and \\ D.H. MORGAN \\ The Royal Observatory, Blackford Hill, Edinburgh
}

Recently a number of new planetary nebulae (PN) have been found in the outer field of the LMC using deep objective-prism J plates taken with a Schmidt Telescope at the AAO. For a number of these PN, we have obtained spectrophotometry on the $2.3 \mathrm{~m} \mathrm{ATT}$ and for a sub-sample, determined the radial velocities using the Echelle Spectrograph of the $2.3 \mathrm{~m}$ Telescope. All this data is to appear in the ApJ.

Since the PN are concentrated in the outer portions of the face of the LMC, they allow an improved determination of a rotation solution given by:

$$
V(\theta, r)=V_{m}(r)\left(1 \pm\left(\tan \left(\theta-\theta_{0}\right) \sec i\right)^{2}\right)^{-0.5}+V_{0}(0 \leq \theta \leq 2 \pi)
$$

where $V(\theta, r)$ is the rotational velocity projected onto the line of sight at position angle $\theta$ and at radial coordinate $r$. The rotation solutions derived are as follow:

\begin{tabular}{lrrr}
\hline Sample & No. Objects & $V_{0}(\mathrm{~km} / \mathrm{s})$ & $\theta_{0}$ \\
\hline This sample & 11 & 40 & 164 \\
All PN outside 4deg. & 30 & 46 & 170 \\
All PN in the LMC & 106 & 42 & 168 \\
\hline
\end{tabular}

The faint PN fall into three distinct groups: first Type I PN with high electron temperatures, very strong lines in HeII, [NII], and often, [Ne V]; second, optically thick PN displaying unusually strong [OII] lines for a $\mathrm{PN}$; and third, optically thin PN of intermediate class. Optically thin objects are comparatively rare in this sample, whereas one would have expected a large number among the faint PN. The optically thick non Type I objects are mainly of excitation class 2-6. However, this appears to be due mainly to low ionisation parameter, rather than to cool nuclei. The predominance of faint optically thick PN appear to be a result of the evolution of central star. Normally a PN ionises more and more of the surrounding gas as it expands, thanks to the decreasing density. If a nebula becomes optically thin, its $H \beta$ luminosity will decrease as $t^{-3}$ and it will rapidly become very faint as a PN. Suppose now that the PN is optically thick when it commences its rapid fading. Models of the evolution of PNN along the fading tracks show that the fading of the central star is likely to be so rapid $\sim t^{-4 t o 5}$ as to overcome the effects of the nebular expansion, and thus the nebula may actually start to recombine until the luminosity of the central star falls below $\sim 100 \mathrm{~L}_{\odot}$. After this the rate of the decline slows considerably $\left(\sim t^{-1}\right)$, and the nebula becomes optically thin to the ionising radiation. All the Type I PN were found to lie near or to the high mass side of the $2.5 \mathrm{M}_{\odot}$ track, confirming that these objects originate from high mass precursors. 